\title{
Determination of the Elastic Constants of Gold Nanorods Produced by Seed
}

\section{Mediated Growth}

Min Hu, ${ }^{*}$ Patrick Hillyard, ${ }^{*}$ Gregory V. Hartland, ${ }^{*} \dagger$ Thomas Kosel, ${ }^{\ddagger}$ Jorge Perez-Juste ${ }^{\S}$ and Paul Mulvaney ${ }^{\S}$

Department of Chemistry and Biochemistry

University of Notre Dame, Notre Dame, Indiana 46556-5670

Department of Electrical Engineering

275 Fitzpatrick Hall, University of Notre Dame, Notre Dame, Notre Dame, IN 46556

School of Chemistry University of Melbourne, Australia

* Department of Chemistry and Biochemistry, University of Notre Dame

${ }^{\dagger}$ Corresponding author

* Department of Electrical Engineering, University of Notre Dame

${ }^{\S}$ School of Chemistry University of Melbourne 


\section{Supporting Information}

The naorods in our experiments were synthesized using the seed mediated growth technique developed by Murphy and co-workers, ${ }^{1,2}$ and the El-Sayed group. ${ }^{3}$ Representative high-resolution transmission electron microscope (HRTEM) images and the corresponding selected area electron diffraction (SAED) patterns from gold nanorods used in our experiments are presented in Figures S-1 and S-2. (The nanorod in S-1 is also discussed in the main text of the paper.) The HRTEM images and diffraction patterns are consistent with the 5-fold twinned model discussed in detail in Refs. [4,5,6,7,8,9]. This model is adopted from a model developed for decahedral particles. ${ }^{10}$ Decahedral particles are constructed from five distorted tetrahedra that each contain four $\{111\}$ faces. The tetrahedra share $\{111\}$ faces along the common [110] axis. Rods can be thought of as being generated by adding (110) planes to the center of the particles (clearly this is not how they actually grow in solution). This gives a structure with $\{100\}$ facets on the sides and $\{111\}$ facets at the ends, and a [110] growth direction - as shown in scheme A below.

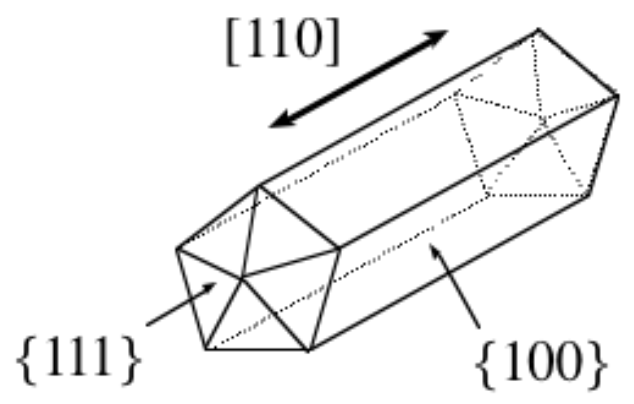

Scheme A: Model for nanorods produced by seed mediated growth. 
Nanorods of cubic materials with this structure can give two types of easily recognizable diffraction patterns, depending on the orientation of the rod with respect to the electron beam. ${ }^{4,5,6,7,8,9}$ Specifically, these arise from the superposition of either the $<110>$ and $<111>$, or the $<100>$ and $<112>$ crystallographic zones. Figure S-1 shows a rod where diffraction from the $<110>$ and $<111>$ crystallographic zones was observed. The outlined hexagon of $\{220\}$ spots in the diffraction pattern arises from the $<111>$ zone, and the spots inside this can be assigned to diffraction from the $<110>$ zone. The $<110>$ direction along the rod axis corresponds to the horizontal $\{220\}$ vector in the diffraction pattern. The HRTEM image in Figure S-1 shows both sets of $\{111\}$ lattice fringes on the lower half of the rod, as is expected for this rod orientation. ${ }^{7}$ The line that runs down the axis of the rod shows the intersection of the twin planes.

Figure S-2 shows a rod where the diffraction pattern is a superposition of the $<112>$ and $<100>$ crystallographic zones, imperfectly aligned along the zone axes. The rectangle characteristic of the $<112>$ zone (with $\{113\}$ corners), and half of the square from the $<100>$ zone (with $\{220\}$ corners) are outlined in the diffraction pattern. The HRTEM image in Figure S-2 shows continuous $\{111\}$ lattice fringes that run down the axis of the rod, again consistent with the diffraction conditions for this rod., ${ }^{4,5,6,7,8,9}$ These fringes are modulated into wider stripes in the center of the rod due to double diffraction - see Refs. [4,5,7] and the multislice calculations in Ref. [5]. 

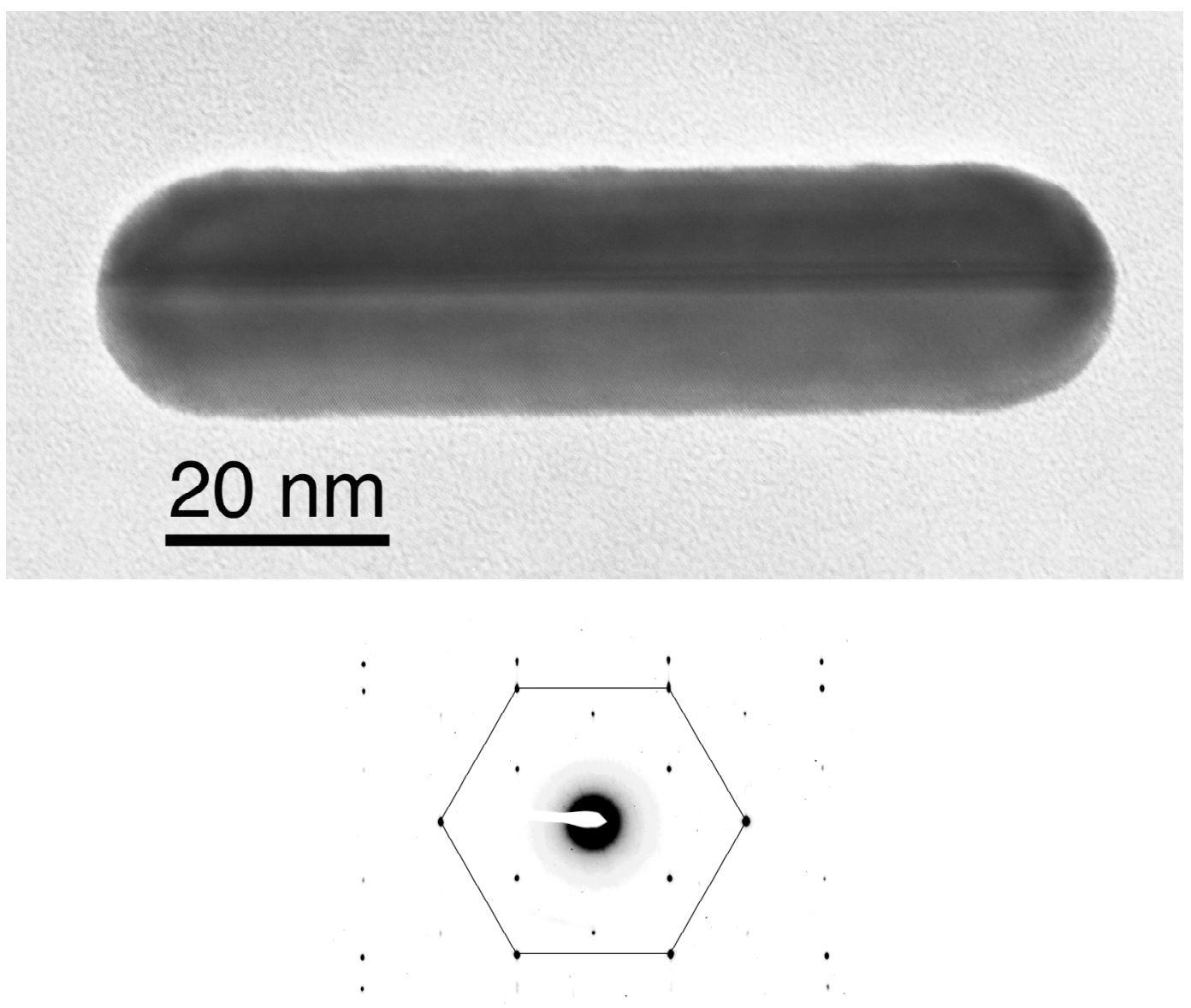

Figure S-1: HRTEM image (top) and corresponding electron diffraction pattern

(bottom) from a nanorod orientated to give diffraction from the $<110>$ and $<111>$ crystallographic zones. 

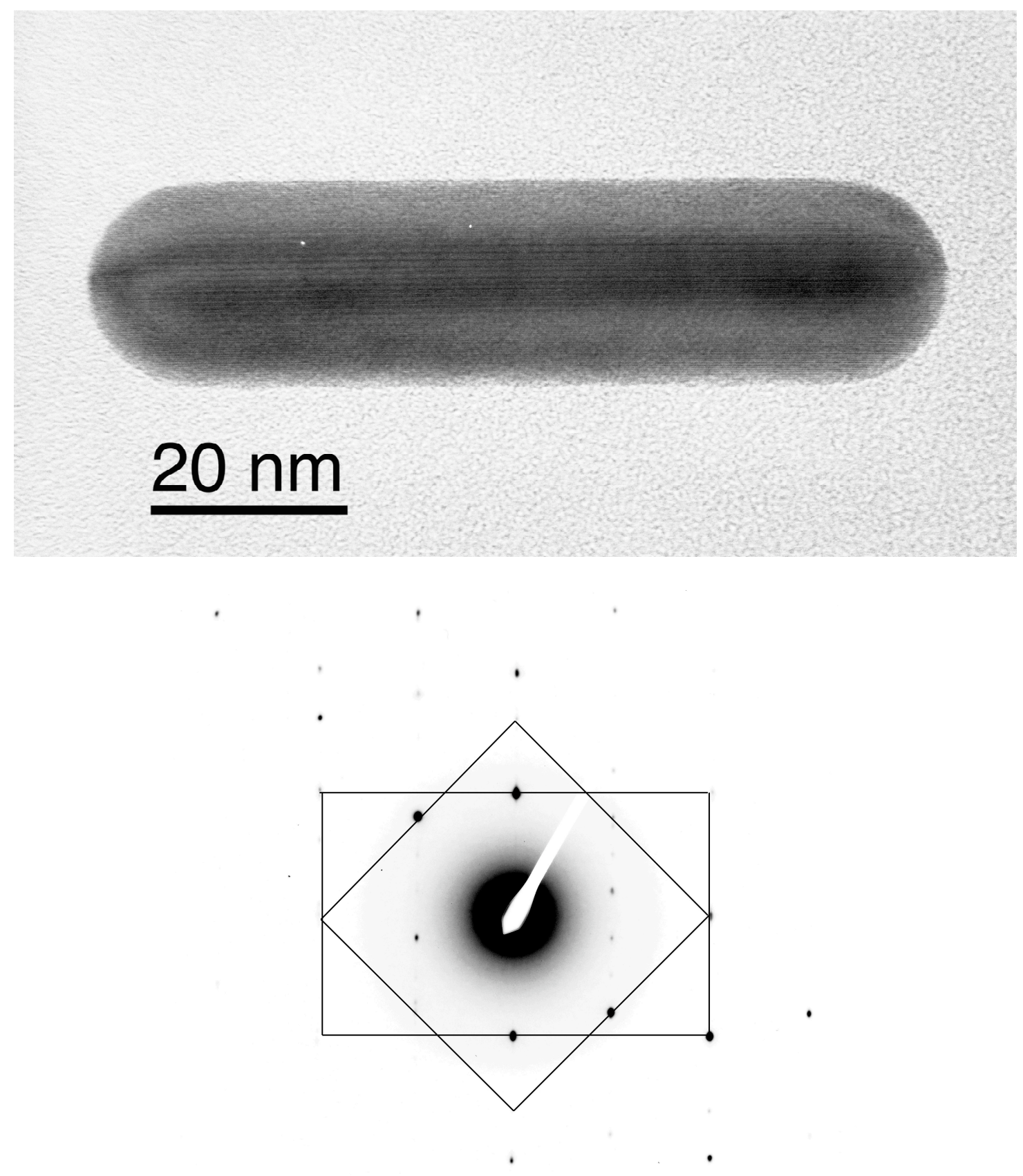

Figure S-1: HRTEM image (top) and corresponding electron diffraction pattern (bottom) from a nanorod orientated to give diffraction from the $<100>$ and $<112>$ crystallographic zones. 
These images and the associated electron diffraction patterns demonstrate that the nanorods in our samples (which were produced using the seed mediated growth technique), have the $\{100\}$ side facets and growth along the [110] direction characteristic of the 5-fold twinned structure outlined in Scheme A. We note, however, that the nanorod ends appear quite rounded, and do not exhibit $\{111\}$ end facets.

\section{References:}

1 Jana N. R.; Gearheart L.; Murphy C. J. J. Phys. Chem. B 2001, 105, 4065.

2 Jana N. R.; Gearheart L.; Murphy C. J. Adv. Mater. 2001, 13, 1389.

3 Nikoobakht, B.; El-Sayed, M. A. Chem. Mater. 2003, 15, 1957.

4 Nepijko, S. A.; Ievlev, D. M.; Schulze, W.; Urban, J.; Ertl, G.; Chem. Phys. Chem., 2000, 140 .

5 Lisiecki, I.; Filankembo, A.; Sack-Kongehl, H.; Weiss, K.; Pileni, M.-P.; Urban, J. Phys. Rev. B 2000, 61, 4968.

6 Hofmeister, H.; Nepijko, S. A.; Ievlev, D. M.; Schulze, W.; Ertl, G.; J. Cryst. Growth, 2002, 234, 773 .

7 Johnson, C. J.; Dujardin, E.; Davis, S. A.; Murphy, C. J.; Mann, S. J. Mater. Chem. 2002, 12, 1765 .

8 Sun, Y. G.; Mayers, B.; Herricks, T.; Xia, Y. N. Nano Lett. 2003, 3, 955.

9 Chen, H.; Gao, Y.; Zhang, H.; Liu, L.; Yu, H.; Tian, H.; Xie, S.; Li, J. J. Phys. Chem. B 2004, 108, 12038.

10 Marks, L. D. Rep. Prog. Phys. 1994, 57, 603. 
